\title{
Introduction to the Vol. 45, No. 1, 2018
}

\author{
Maomi Ueno ${ }^{1}$
}

Published online: 19 March 2018

(C) The Behaviormetric Society 2018

Welcome to the Vol. 45, No. 1, 2018 of Behaviormetrika. In this issue, we have the following nine original papers and one short note.

The original paper "Asymmetrical triadic relationship based on the structural difficulty: A Bayesian approach for balance theory', by Koji E. Kosugi (2018) addresses the magnitude estimation of the cognitive load when a person perceives asymmetric social relations. In this study, since a balanced state is defined by structural complexity, an experimental hypothesis was set whereby asymmetric social relationships have different difficulty levels depending on structural complexity. The balanced state of an asymmetric relation as structural difficulty is formally derived from the eigenvalue structure of a Hermitian matrix. Asymmetric triadic relations are modeled as featuring three kinds of structural difficulties according to the eigenvalue decomposition of the Hermitian matrix and pattern specific difficulties. The differences among the structural difficulties were not sufficiently significant to exceed pattern-specific difficulties, but the Bayes factor of the informational hypothesis of this research yielded positive effects.

The original paper "Estimation of unobserved dynamics of individual partisanship: a Bayesian approach', by Tomohito Okabe and Daisuke Nogiwa (2018) proposes a Bayesian estimation of Markov dynamics of individual-level partisanship with repeated cross-section data in which the history of respondents' choice of favored party cannot be observed. The proposed method identifies individual heterogeneities that affect transitional patterns of partisanship, and replicates the dynamic patterns of individual partisan mobility. Using the proposed method with American survey data, the study shows that age, education and race significantly influence partisan dynamics among Americans over a three-decade period from 1972.

Maomi Ueno

ueno@ai.is.uec.ac.jp

1 University of Electro-Communications, Tokyo, Japan 
The original paper "Economic voting using egotropic evaluation as an information cue: how absolute and relative income affects socio-economic evaluations and retrospection', by Hanako Ohmura (2018), using an internet survey, examines how perceptions of economic disparity affect income tax rate preferences founded on absolute income. This paper then tested whether egotropic and sociotropic evaluations separately affect voters' retrospection on government, or if the easier egotropic evaluation functions as an information cue for the more difficult sociotropic evaluation. The results demonstrated the following: (1) those who perceive economic inequality to exist tend to be punitive toward voters in other income brackets; (2) this punitive tendency is stronger among higher and middle earners; and (3) for the middle earners, absolute income status and for the higher earners, perception of economic inequality mediates the effect of sociotropic evaluations and ultimately influences government approval.

The original paper "Weighted squared distance for two-deck randomized response model" by Augustus Jayaraj, Oluseun Odumade, Stephen Sedory, and Sarjinder Singh (2018) proposes a new weighted squared distance while minimizing a distance between the true proportions and the observed proportions of (Yes, Yes), (Yes, No), (No, Yes) and (No, No) answers in the setup of Odumade and Singh (Commun Stat Theory Methods 38: 439-446, 2009) model. The resultant estimator is shown to be an unbiased estimator of the proportion of the sensitive attribute of interest in a population and has smaller variance than the estimator of Odumade and Singh (Commun Stat Theory Methods 38: 439-446, 2009) with the same protection of the respondents.

The original paper "Simultaneous canonical correlation analysis with invariant canonical loadings' by Fei Gu and Hao Wu (2018) addresses a restrictive canonical correlation analysis. In canonical correlation analysis (CCA), the substantive interpretations of the canonical variates are of primary interest to the applied researchers. However, there are two different interpretive approaches used by different researchers-the weight-based approach and the loading-based approach, of which the latter is favored by the majority of researchers in practice. For those who choose the loading-based approach and apply CCA simultaneously to multiple samples, they may wish to test the invariance of the canonical loadings. In this paper, three covariance structure models are defined for CCA. In particular, the first model (i.e., the CCA-W model) corresponds directly with regular CCA, including the canonical correlations and canonical weights as the parameters, while the third model (i.e., the CCA-L model) is in alignment with the loading-based interpretive approach, including the canonical correlations and canonical loadings as the parameters. The CCA-L model is further extended to the unrestricted and restricted SCCA-L models, of which the latter allows one to test the invariance of the canonical loadings. A real example drawn from the sociological literature is provided to illustrate the restrictive SCCA-L model, and some strategies to calculate good starting values for the restrictive SCCA-L model are discussed.

The original paper "Social constructivist approach of motivation: social media messages recommendation system' by Sébastien Louvigné, Masaki Uto, Yoshihiro Kato, and Takatoshi Ishii (2018) develops a learning system to enhance learning motivation. Contemporary learning theories and their implementations associated 
with information and communication technologies increasingly integrate social constructivist approaches to assist and facilitate the construction of knowledge. Social constructivism also highlights the important role of culture, learning attitude and behavior in the cognitive process. Modern e-learning systems need to include these psychological aspects in addition to knowledge construction to connect with longstanding pedagogical issues such as the decrease and lack of motivation for education. Current Social Networking Services (SNS) provide a platform where peers can express their passion, emotion, and motivation toward learning. Therefore, this research utilizes this platform to recommend motivational contents from peers for learning motivation enhancement (i.e., learners' perception of their goal and purpose for learning). The proposed system consists of an SNS platform for learners to (1) express and evaluate their own goals for learning, (2) observe motivational messages from peers recommended from an LDA-based (latent Dirichlet allocation) model, and (3) evaluate their perceptions on motivational and psychological attributes. The LDA-based model recommends messages expressing diverse purposes for a shared goal by maximizing the topic divergence of Twitter messages. Learners' self-evaluations show the positive and significant impact of observing diverse learning purposes from peers on intrinsic motivational attributes such as goal specificity, attainability, and on the confidence to achieve the desired outcome.

The original paper "Comparisons among several consistent estimators of structural equation models" by Yoshio Takane and Heungsun Hwang (2018) compares several consistent estimators of structural equation models. With the advent of consistent partial least squares (PLSc), an interest has surged in comparing the quality of various estimation methods in structural equation models. Of particular interest are, beside PLSc, Bentler's non-iterative confirmatory factor analysis, Hägglund's instrumental variable (IV) estimation method, and Ihara-Kano's noniterative uniqueness estimation method. All of these methods yield consistent estimates of parameters in measurement models (factor loadings and unique variances), but require additional steps to estimate parameters in structural model covariances among latent variables (LVs) and path coefficients. These additional steps typically involve calculating LV scores, either correlating them or applying regression analysis, and correcting possible "biases" incurred by the use of LV scores as proxies of true LVs. This paper conducts a Monte Carlo study to evaluate parameter recovery capabilities of the above LV extraction methods in conjunction with subsequent LV score construction and bias correction methods. We also compare these methods against more conventional estimation methods, such as the full least squares and maximum likelihood methods that estimate parameters in both measurement and structural models simultaneously. In addition, this paper examines three methods of estimating standard errors (SEs) of estimated parameters from a single dataset, the bootstrap method, ordinary least squares regression, and the inverse Hessian method. The SEs are important in assessing the reliability of parameter estimates and in testing their significance. It was found that Hägglund's method used to extract one LV at a time from each block of observed variables, combined with Croon's bias correction method, worked best in both parameter recovery and resistance to improper solutions, and that the bootstrap method provided the most accurate estimates of SEs. 
The original paper "Large-scale estimation in Rasch models: asymptotic results, approximations of the variance-covariance matrix of item parameters, and divide-and-conquer estimation', by Patrick Mair and Helmut Strasser (2018) elaborates on the asymptotics of conditional maximum-likelihood estimates for an increasing number of items, important for modern data settings where a large number of items need to be scaled. Using approximations of the variance-covariance matrix based on Edgeworth expansions, the problem is studied theoretically as well as computationally. In a subsequent step, these results are used to split the large-scale estimation problem into smaller sub-problems involving blocks of items. These item blocks are estimated separately from each other, and finally, merged back into the full parameter vector (divide and conquer). By means of simulation studies, and in conjunction with the asymptotic results, it was found that block sizes in the range of 30-40 items approximate the full-scale estimators with a negligible loss in precision. It is also shown how varying block sizes affect the running time needed to fit the model.

The short note "Direction of comparison effect and consumer research: from general to specific implications", by Shuzo Abe and Yoshiyuki Okuse (2018) tries to move toward the application of the direction of comparison effect (DCE) in marketing. When two alternatives are compared, the one that becomes the focus of comparison has a relative advantage (resp., disadvantage) when two alternatives share positive (resp., negative) unique features. This DCE has been found to manifest clearly when three conditions are met: when information is retrieved from memory rather than being externally presented, when a consumer has low product involvement but a high need for cognition, and when the attribute set size is large. The authors argue that at least two additional conditions must be met when applying the DCE in marketing: (1) the presence of unique features of market offerings and (2) the absence of situational factors such as commitment to an alternative. Experimental data on choice behavior regarding business hotels provide support for these two conditions. Also, in discussing the results, it has become clear that unnoticed DCE bias should be carefully canceled out in surveys by designing questionnaires to ensure that each alternative randomly becomes the focal alternative.

\section{References}

Abe S, Okuse Y (2018) Direction of comparison effect and consumer research: from general to specific implications. Behaviormetrika 45(1). https://doi.org/10.1007/s41237-017-0039-3

Gu F, Wu H (2018) Simultaneous canonical correlation analysis with invariant canonical loadings. Behaviormetrika 45(1). https://doi.org/10.1007/s41237-017-0042-8

Jayaraj A, Odumade O, Sedory S, Singh S (2018) Weighted squared distance for two-deck randomized response model. Behaviormetrika 45(1). https://doi.org/10.1007/s41237-017-0041-9

Kosugi K (2018) Asymmetrical triadic relationship based on the structural difficulty: A Bayesian approach for balance theory. Behaviormetrika 45(1). https://doi.org/10.1007/s41237-017-0033-9

Louvigné S, Uto M, Kato Y, Ishii T (2018) Social constructivist approach of motivation: social media messages recommendation system. Behaviormetrika 45(1). https://doi.org/10.1007/s4123 7-017-0043-7 
Mair P, Strasser H (2018) Large-scale estimation in Rasch models: asymptotic results, approximations of the variance-covariance matrix of item parameters, and divide-and-conquer estimation. Behaviormetrika 45(1). https://doi.org/10.1007/s41237-018-0046-z

Ohmura H (2018) Economic voting using egotropic evaluation as an information cue: how absolute and relative income affects socio-economic evaluations and retrospection. Behaviormetrika 45(1). https ://doi.org/10.1007/s41237-017-0040-x

Okabe T, Nogiwa D (2018) Estimation of unobserved dynamics of individual partisanship: a Bayesian approach. Behaviormetrika 45(1). https://doi.org/10.1007/s41237-017-0034-8

Takane Y, Hwang H (2018) Comparisons among several consistent estimators of structural equation models. Behaviormetrika 45(1). https://doi.org/10.1007/s41237-017-0045-5 